\title{
Quality assurance in IMRT: Importance of the transmission through the jaws for an accurate calculation of absolute doses and relative distributions
}

\author{
Juan Diego Azcona ${ }^{a)}$ \\ Department of Oncology, Division of Radiation Physics, Clínica Universitaria de Navarra, Avda. Pío XII, \\ 36, 31008 Pamplona, Navarra, Spain \\ R. Alfredo C. Siochi \\ Siemens Medical Systems, Oncology Care Systems, Apex, North Carolina 27502 \\ Ignacio Azinovic \\ Department of Oncology, Division of Radiotherapy, Clínica Universitaria de Navarra, Avda. Pío XII, \\ 36, 31008 Pamplona, Navarra, Spain
}

(Received 3 May 2001; accepted for publication 8 November 2001; published 8 February 2002)

\begin{abstract}
The goal of IMRT is to achieve an isodose distribution conformed to the tumor while avoiding the organs at risk. For these tasks several gantry angles are selected, each one containing a series of different leaf configurations for the multileaf collimator (MLC) (segments). Verifying the relative distributions as well as the absolute doses is an important step for quality assurance issues. We have observed that an accurate modeling of the transmission of the primary x-ray fluence through the jaws and MLC as well as the head scatter is crucial for a precise calculation of relative doses and monitor units. Also, an inaccurate calculation of the output factor for small size segments can lead to important differences in the absolute dose for points under these segments. Incorrect models could lead to systematic errors of around 5\% to $10 \%$ in the calculated monitor units and a shift in the isodose curves. (C) 2002 American Association of Physicists in Medicine.
\end{abstract}

[DOI: $10.1118 / 1.1446110]$

Key words: multileaf collimator, intensity modulation, dosimetry, inverse planning, IMRT treatment plan verification

\section{INTRODUCTION}

IMRT has been implemented recently at the Clínica Universitaria de Navarra, Pamplona, Spain. Treatments are performed using the "step-and-shoot" technique, ${ }^{1-3}$ where several gantry angles are selected depending upon tumor location, each one containing a series of different static configurations of the MLC leaves (segments), which have the effect of modifying the fluence map of each field according to a set of requirements previously introduced by the user. The sum of the different contributions of each beam to the dose over the patient's anatomy results in an optimal dose distribution, where the prescription isodose surfaces are conformed to the tumor's shape while avoiding the organs at risk.

In this work, we describe the differences between measured and calculated absolute doses and isodoses we have observed when carrying out the verification procedure, due to an incorrect estimation of the head scatter and the transmission through the jaws during the calculations by the Treatment Planning System. Also, the incorrect modeling of the output factors for small fields affects the calculations of the absolute dose for points under the primary beam for those segments.

\section{MATERIAL AND METHOD}

The equipment available at our institution consists of a Siemens Mevatron Primus linear accelerator with a multileaf collimator, which replaces the jaws in the $X$ direction. We have been using $15 \mathrm{MV}$ photon beams for IMRT treatments. The software used for the optimization of dose distributions and calculations was MDS Nordion TMS version 5.1 B (Helax $A B)$. Fields were exported from the treatment planning system to the linac via the LANTIS network and the treatment was performed with the Prime View module, which allows the user to deliver an automated sequence of segments.

The verification of dose distributions is a key point for the quality assurance of IMRT treatments. We followed DKFZ Heidelberg's verification procedure ("IMRT Workshop," DKFZ Heidelberg, 25-28 November 1999, Handbook) to compare the doses calculated by the TMS system with the measurements taken on our linac for all the clinical cases we performed. ${ }^{4}$

\section{A. Cases to study}

We present data for five different clinical cases and also for four test cases supplied to us by MDS Nordion (Helax AB). All clinical cases are pelvic tumors. They consist of a number of gantry positions between five and seven. The field sizes that enclose all the segments are $7 \times 11 \mathrm{~cm}^{2}$, $8 \times 10 \mathrm{~cm}^{2}, 17 \times 15 \mathrm{~cm}^{2}, 14 \times 18 \mathrm{~cm}^{2}$, and $5 \times 7 \mathrm{~cm}^{2}$, while the number of segments for each case are $36,34,44,65$, and 28 , respectively. These fields contain some small size segments. The errors observed in these cases are considered to be the sum of the errors in the modeling of the transmission, 
head scatter and small size segments output factors. The measurements of the absolute doses were taken at low gradient points with a PTW 31002 ionization chamber, with a volume of $0.125 \mathrm{~cm}^{3}$, and a PTW Unidos E electrometer. The relative distributions have been obtained using radiographic film.

For the test cases we have taken several profiles in the crossplane direction as well as output factors. Each test case consists of one single gantry position with $10,9,7$, and 16 segments, respectively. The fields which enclose all the segments are $18 \times 18 \mathrm{~cm}^{2}, 6 \times 25 \mathrm{~cm}^{2}, 12 \times 12 \mathrm{~cm}^{2}$, and $12 \times 12$ $\mathrm{cm}^{2}$, respectively. Results are displayed comparing measurements to calculations with and without the fudge factor. Profiles have been taken with Scanditronix' Linear Detector Array 25, and output factors were measured with a PTW 31002 ionization chamber $0.125 \mathrm{~cm}^{3}$ volume. These test cases do not contain any small size segment, so the errors observed here are considered to be related only to incorrect models for transmission and head scatter.

Also we have measured several small fields (with an area less than 9 sq. $\mathrm{cm}$ ) output factors. For this task we have used a Scanditronix stereotactic diode, with a detector diameter of $0.6 \mathrm{~mm} \pm 0.1 \mathrm{~mm}$. We have compared these measured output factors with the calculated ones. Calculations and measurements for these small size fields have been taken with the upper jaws closed to the limit of the field and with the upper jaws opened $11 \mathrm{~cm}$ width, so the magnitudes of the different errors in one field can be seen.

\section{B. The fudge factor}

The fudge factor is a parameter that adjusts the transmission of the primary $x$-ray fluence through the jaws. ${ }^{5}$ In the first two clinical cases studied, the calculations were taken by considering this factor set to 1.015 . (This is interpreted as a transmission of $1.5 \%$ to locations underneath the MLC leaves.) The last three clinical cases were calculated by removing the effects of transmission, i.e., setting the fudge factor equal to 1.000 . The test cases have been calculated separately with and without the fudge factor.

\section{RESULTS AND DISCUSSION}

\section{A. Clinical cases}

For the first two cases an important discrepancy between absolute doses at single points calculated by TMS and the corresponding measurements was observed. The results are shown in Table I.

After these results were observed, the fudge factor, used by the treatment planning system for calculations, was modified. The previous value for this factor was 1.015, and it was changed to 1.000 as recommended by MDS Nordion (Helax $\mathrm{AB})$. This factor adjusts the transmission of the primary $\mathrm{x}$-ray fluence through the jaws. Setting the fudge factor to 1.000 removes the contributions due to the transmission. After this modification was done, the agreement between the calculations and the measurements of the absolute dose clearly improved (Table II), although there was still a re-
TABLE I. Absolute doses measured and compared with calculations with the fudge factor. Case 1 is a six-field plan and case 2 is a five-field plan.

\begin{tabular}{lcccc}
\hline No. Case & Point & $\begin{array}{c}\text { Measured dose } \\
(\mathrm{cGy})\end{array}$ & $\begin{array}{c}\text { Calculated dose } \\
(\mathrm{cGy})\end{array}$ & \% Difference \\
\hline Case 1 & 1 (Isocenter) & 206.4 & 210.5 & 2.0 \\
& 2 & 199.3 & 207.2 & 4.0 \\
& 3 & 185.2 & 206.1 & 11.3 \\
Case 2 & 1 (Isocenter) & 196.9 & 208.6 & 5.9 \\
& 2 & 185.7 & 189.5 & 4.7 \\
& 3 & 185.3 & 199.0 & 7.2 \\
& 4 & 183.4 & 201.8 & 8.9 \\
\hline \hline
\end{tabular}

sidual error. The explanation for this error is as follows: TMS sends the MLC configurations to LANTIS properly, but for the final calculations, after the optimization process, it considers the jaws to be at a fixed position for all segments in a beam. This position is defined by the smallest opening that exposes all the segments in a beam [private communication, MDS-Nordion (Helax AB)]. In the Siemens Mevatron Primus, the jaws are moved for each segment to be set at the limit of the open field for that segment, so this leads to a difference in the transmission of the primary $x$-ray fluence (with higher calculated values since the jaws decrease transmission), as well as a difference in the scattering in air from the linac head. [Analytic models for calculating the head scatter in TMS (Dose Formalism and Models in Helax-TMS, Helax AB 1998) have been developed by Ahnesjo ${ }^{6,7}$ and Ahnesjö et al. $\left.{ }^{8}\right]$ Both transmission and scattering were overestimated before the change in the fudge factor, so the calculated dose was always greater than the measured one. With the removal of the fudge factor, transmission is underestimated while scattering remains overestimated. Removing the fudge factor has corrected part of the first of these errors, whereas the second one remains unchanged. In most of the situations we have observed, the total dose is still overestimated (Table II). This error is more important for the calculation of absolute doses (or monitor units) than for relative distributions, although these are also affected by the error. Also, as these fields contain small size segments, part of the error could be related to an incorrect modeling of the output factors for these segments. Figures 1 and 2 show the planned case and the overlay of the calculated and measured isodoses, respectively. Figure 3 zooms in on the area of dis-

TABLE II. Absolute doses measured and compared with calculations without the fudge factor. Case 3 is a five-field plan and case 4 is a seven-field plan.

\begin{tabular}{lcccc}
\hline \hline No. Case & Point & $\begin{array}{c}\text { Measured dose } \\
(\mathrm{cGy})\end{array}$ & $\begin{array}{c}\text { Calculated dose } \\
(\mathrm{cGy})\end{array}$ & \% Difference \\
\hline Case 3 & 1 (Isocenter) & 197.0 & 200.0 & 1.5 \\
& 2 & 207.6 & 212.0 & 2.1 \\
& 3 & 201.0 & 205.6 & 2.3 \\
Case 4 & 4 & 197.4 & 208.0 & 5.4 \\
& 1 (Isocenter) & 219.5 & 222.0 & 1.2 \\
& 2 & 217.2 & 220.0 & 1.3 \\
& 3 & 211.5 & 214.4 & 1.3 \\
\hline \hline
\end{tabular}




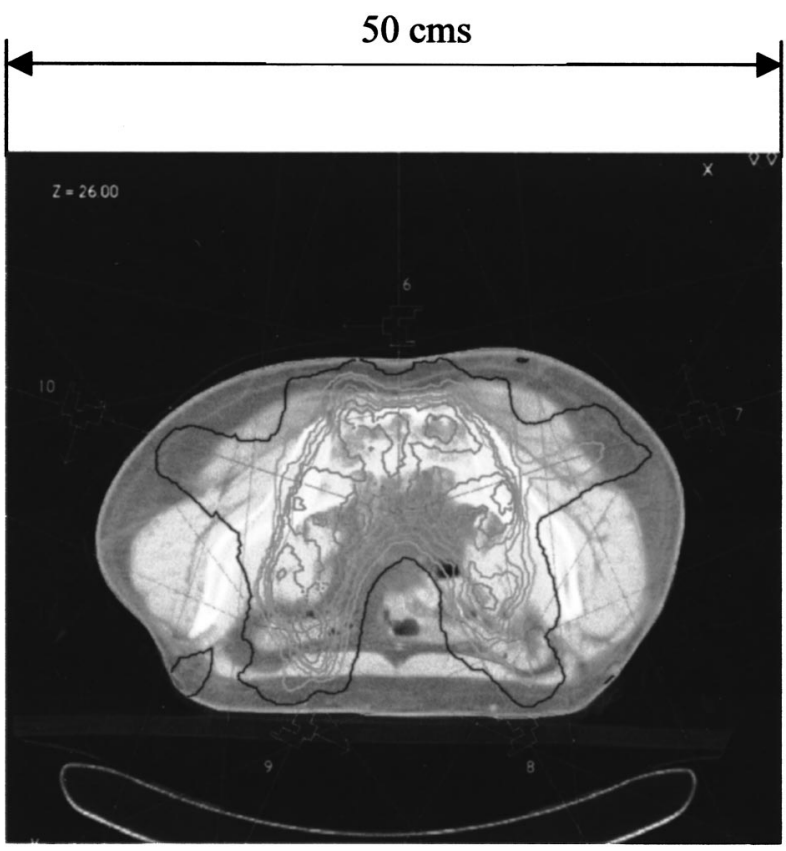

FIG. 1. IMRT horsehoe dosimetry. Isodoses are $10 \%, 50 \%, 70 \%, 80 \%, 85 \%$, $90 \%, 100 \%, 105 \%$

agreement that is due mainly to the AP field and shows that the calculated isodoses are shifted with respect to the measured ones, because the field is blocked in that area (the beam is passing right through critical structures). The $90 \%$ and $95 \%$ lines show that the calculations overestimate scatter, while the $60 \%$ line and part of the $80 \%$ line directly under the dip in the $60 \%$ area show that the calculations underestimate leakage due to the removal of the fudge factor.

We have calculated and measured another case without the fudge factor. Here the differences are greater than for cases 3 and 4 (Table III). The reason is that for this particular

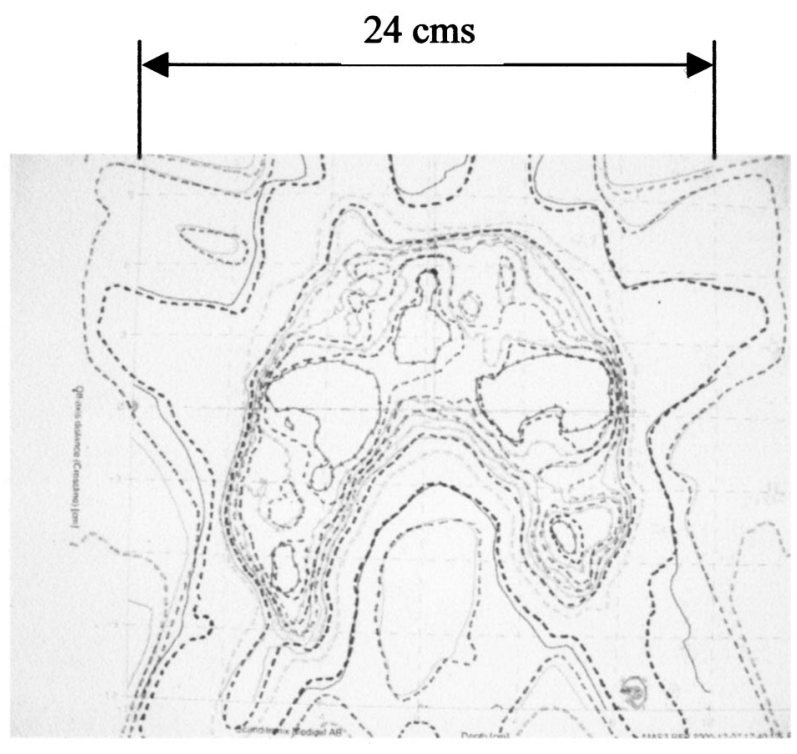

FIG. 2. Verification of IMRT distribution. Continuous lines are measured isodoses and dotted lines are calculated ones. Isodoses are 20\%, 40\%, 60\%, $80 \%, 90 \%, 95 \%, 100 \%, 105 \%, 110 \%$.

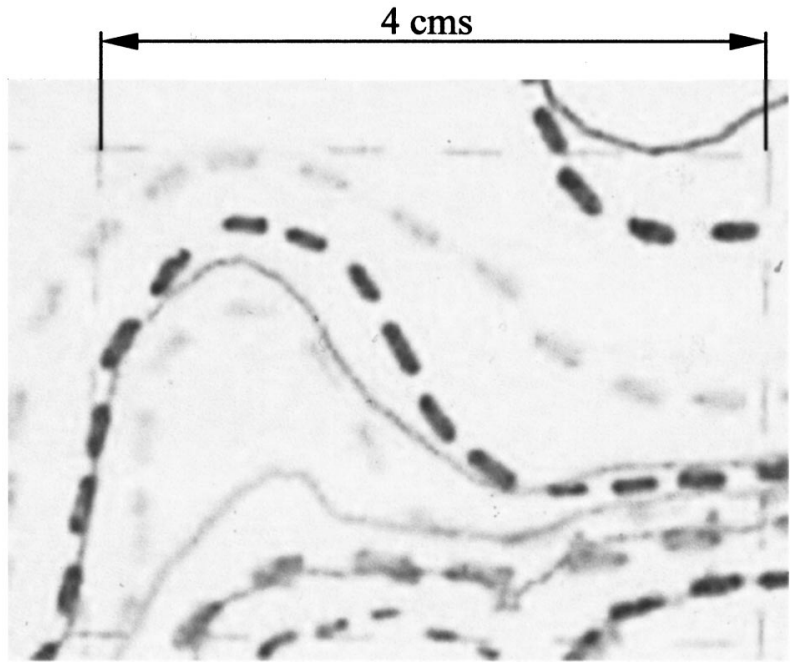

FIG. 3. Zoomed-in view of the shifts in the $60 \%, 80 \%, 90 \%$, and $95 \%$ isodose lines due to underestimating the leakage $(60 \%$ and $80 \%)$ and overestimating the head scatter (90\% and 95\%), isodose lines are the same as in Fig. 2.

case, fields are much smaller than for the other cases. For case 3 , all segments are confined between rectangular fields of $17 \times 15 \mathrm{~cm}^{2}$, and for case 4 , within $14 \times 18 \mathrm{~cm}^{2}$. For this last case, the field that encompasses all the segments is $5 \times 7 \mathrm{~cm}^{2}$. The differences in the head scatter, and thus the total scatter, between the real and the calculated positions are greater, because the head and the total scatter factor vary more abruptly for small fields. So the error grows as the field size decreases. In addition to this, the ratio of small size segments to the total number is higher than in the other cases, so the error in the output factor for these segments affects the overall error. (For cases 1 and 2, the rectangular fields that expose all the segments are $7 \times 11 \mathrm{~cm}^{2}$ and $8 \times 10$ $\mathrm{cm}^{2}$, respectively.)

\section{B. Test cases}

These fields do not contain any small size segment, so errors are considered to be related only to an inaccurate modeling of the transmission and head scatter. Removing the fudge factor leads to a better agreement between the calculations and the measurements. Without the fudge factor, the difference for the output factors is between $\pm 5 \%$. As the error is more important for absolute doses than for relative distributions, we can more readily observe the improvement in the output factor table (Table IV). In the profiles, the im-

TABLE III. Differences for a small field case without the fudge factor.

\begin{tabular}{lcccc}
\hline \hline No. Case & Point & $\begin{array}{c}\text { Measured dose } \\
(\mathrm{cGy})\end{array}$ & $\begin{array}{c}\text { Calculated dose } \\
(\mathrm{cGy})\end{array}$ & \% Difference \\
\hline Case 5 & 1 (Isocenter) & 184.0 & 189.5 & 2.5 \\
& 2 & 178.1 & 190.6 & 7.0 \\
& 3 & 191.0 & 202.4 & 6.0 \\
& 4 & 188.0 & 197.4 & 5.3 \\
\hline \hline
\end{tabular}


TABLE IV. Comparison between measured and calculated (with and without the fudge factor) output factors for four modulated test cases.

\begin{tabular}{cccccr}
\hline \hline No. Field & Measured OF & Calc. OF fudge & $\begin{array}{r}\text { Diff. } \\
(\%)\end{array}$ & $\begin{array}{r}\text { Calc. OF } \\
\text { no fudge }\end{array}$ & $\begin{array}{r}\text { Diff. } \\
(\%)\end{array}$ \\
\hline 1 & 0.626 & 0.646 & 3.2 & 0.636 & 1.6 \\
2 & 0.571 & 0.619 & 8.4 & 0.589 & 3.2 \\
3 & 0.929 & 0.958 & 3.1 & 0.963 & 3.7 \\
4 & 0.168 & 0.184 & 9.5 & 0.163 & -3.0 \\
\hline
\end{tabular}

provement can be seen for points outside the field (Fig. 4). Also, if the profiles were to be normalized to the maximum point in the field, one would see that areas of lower dose could benefit the most from the removal of the fudge factor, since these areas are actually blocked by the jaws for a much longer period than any of the other areas. Hence, they would have the greatest contributions from indirect radiation, and any corrections made to the calculation of these contributions would affect the total calculated dose in these regions the most.

However, in case 4, the profile calculated without the fudge factor is below the measured one in the central area.

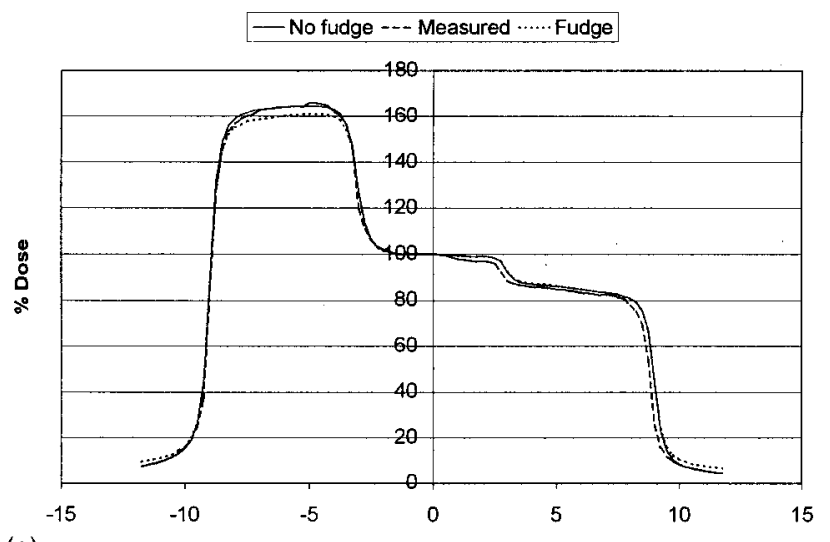

(a)

Axis distance $(\mathrm{cm})$

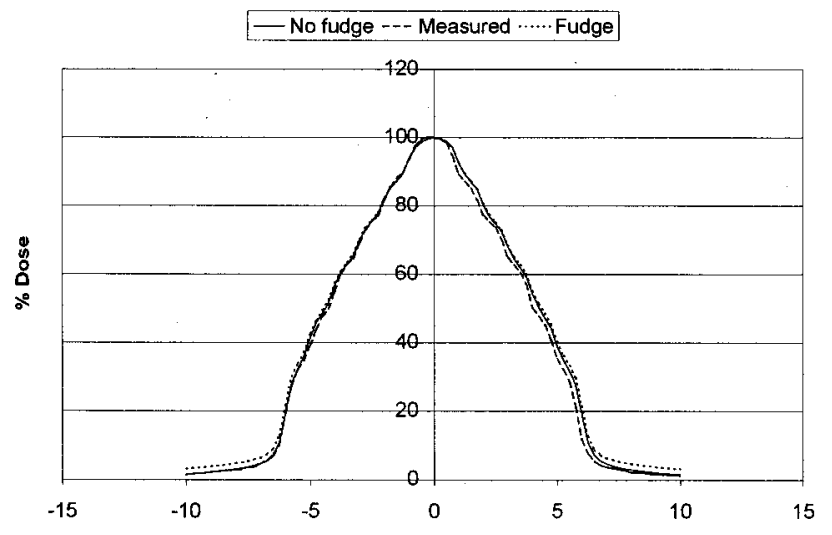

(c)

Axis distance $(\mathrm{cm})$
Removing the fudge factor means not taking transmission into account at all. This field contains 16 segments, 14 of which are blocked at the central axis (which mean $88 \%$ of the weight in fluence for this particular case). The transmission is underestimated, as we can see in the measurement of the output factor. Hence, in this case, the fudge factor should have been reduced but not completely removed. Alternatively, the x-ray transmission through a multileaf collimator could be calculated. A method has been proposed by Chen et $a{ }^{9}{ }^{9}$ In addition to this, the positions of the jaws during calculations should be corrected to improve the dose calculations.

As we can see clearly in case 3, the overestimation of the scattering at the treatment machine head affects the penumbra of the field, which is larger than the measured one. Also, in case four, since the segmentation forces segments to move from the left to the right, the segments contributing to the right-hand side peak will be smaller than those contributing to the one on the left-hand side. Hence, the right-hand side measured peak is shorter than the left-hand side measured peak. Scatter is not being calculated properly since the cal-

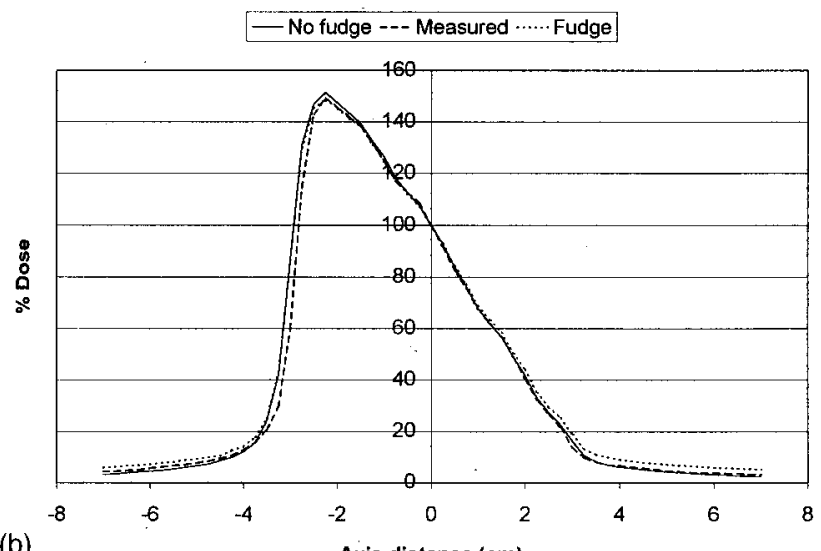

(b)

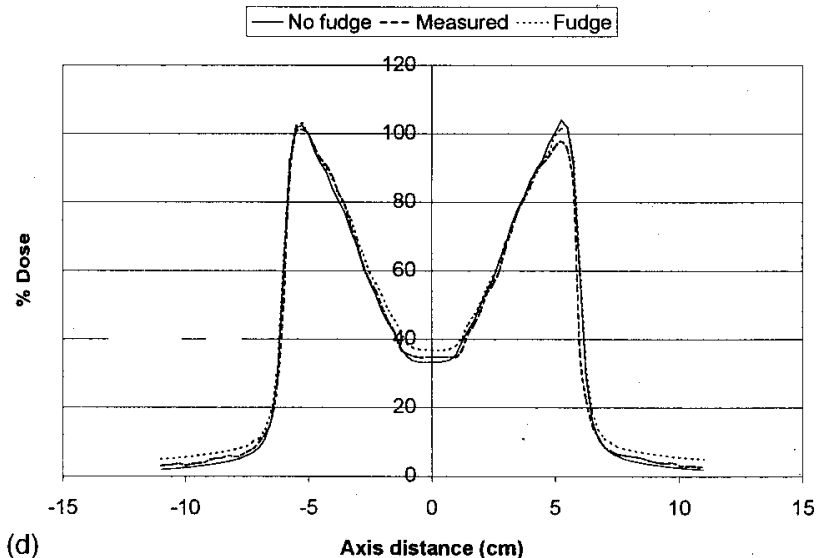

(d)

FIG. 4. Comparison between measurements and calculations with and without the fudge factor for four modulated fields, consisting of (a) 10 segments, (b) 9 segments, (c) 7 segments, and (d) 16 segments. All these profiles have been taken in the crossplane direction at $10 \mathrm{~cm}$ depth. In case 4 we can see the underestimation of the transmission by the calculations without the fudge factor in the central part of the profile, where the field is blocked most of the irradiation time. For all the cases, removing the fudge factor leads to a better agreement between measurements and calculations. In the area outside the field, we can see that calculations with the fudge factor overestimate the dose. 
culations without the fudge factor show the peaks to be of equal heights.

Test case four shows a similar situation to that which occurs for the AP field in Figs. 1-3, and we can see the same effects. In the region of low dose, where the profile dips down, case 4 shows an underestimation due to the lack of leakage; similarly, the area of low dose in the direction of the AP field, where the modulation is attempting to protect critical structures, shows the measured $60 \%$ and $80 \%$ isodose lines to be spread out further from isocenter than the measured ones (i.e., greater measured values), indicating that leakage has been underestimated without the fudge factor. The left-hand side of these isodose lines also show an effect similar to that of the right-hand side of these isodose lines also show an effect similar to that of the right-hand side peak of test case 4, where this time, the calculated isodoses for $90 \%$ and $95 \%$ are spread out further from isocenter than the measured ones (indicating greater calculated values), and in this case we have overestimated scatter in the calculation. This is because the profile for the intensity map of the AP field has two peaks surrounding a dip at the central axis, just as test case 4 does.

Although these individual field errors are significant, it is important to note that, assuming similar field sizes, the order of magnitude of the error is larger for individual fields (Table IV) than for complete plans (Table II), for which errors are averaged. In fact, the deviations due to the AP field in Figs. 2 and 3 are only noticeable in the regions where there are not many other beams contributing to the isodoses. The errors found when examining a complete plan should be considered as of more significance than those seen in individual fields.

\section{Small segments output factors}

Another important source of error which affects only the small segments (less than 9 sq. $\mathrm{cm}$ ) is the modeling of the output factors. Frequently the values for these output factors are overestimated by the TPS, as we can see in Table V. There we present data for small fields measured with the jaws closed to the limit of the leaves and also with the jaws opened to $11 \mathrm{~cm}$ width. The magnitude of the errors due to the overestimation of the output factor and the incorrect positioning of the jaws during the calculations can be compared. Both errors are summed in most situations to give a bigger error, but the global error for a complete field is averaged with the nonsmall segments. However, as the ratio of the number of small segments in a field to the total number of segments increases, so does the error for that field. This error affects mainly the points under the primary $\mathrm{x}$-ray beam of these small size segments.

\section{CONCLUSION}

It is important to use correct models for indirect radiation (scatter and transmission) in the treatment planning system, as their contribution for IMRT plans can be significant. Scatter should be calculated according to an accurate estimation of the head scatter. Thus, the position of the jaws during the calculations should be corrected. Transmission should be
TABLE V. Output factors for several small fields, taken with the jaws opened to $11 \mathrm{~cm}$ width and closed to the limit of the field. Differences for the calculated and measured OFs for these two positions are provided, as well as the overall difference between the calculated field with opened jaws and the measured field with closed jaws. Asymmetric fields have their center at $5 \mathrm{~cm}$ off axis along the $\mathrm{X}$ direction, $0 \mathrm{~cm}$ off axis along the $\mathrm{Y}$ direction. A small offset $(1 \mathrm{~mm})$ in the positioning of the leaves/jaws in a small field could lead to an important error in the OF. For this reason, in this table, the nominal field is the actual radiation field to avoid errors due to an incorrect positioning of the leaves/jaws.

\begin{tabular}{|c|c|c|c|c|c|}
\hline Field (X by $\mathrm{Y})$ & Jaws & Calculated & Measured & $\begin{array}{c}\text { Diff. } \\
\text { Calc-meas } \\
(\%)\end{array}$ & $\begin{array}{c}\text { Overall } \\
\text { diff. } \\
(\%)\end{array}$ \\
\hline \multirow{3}{*}{$\begin{array}{l}\text { Symmetric } 3 \times 3 \\
\mathrm{~cm}^{2}\end{array}$} & Opened & 0.876 & 0.842 & 4.0 & \multirow{3}{*}{5.9} \\
\hline & Closed & 0.869 & 0.827 & 5.1 & \\
\hline & Diff. (\%) & 0.8 & 1.8 & & \\
\hline \multirow{3}{*}{$\begin{array}{l}\text { Symmetric } 1 \times 1 \\
\mathrm{~cm}^{2}\end{array}$} & Opened & 0.645 & 0.598 & 7.9 & \multirow{3}{*}{14.0} \\
\hline & Closed & 0.599 & 0.566 & 5.8 & \\
\hline & Diff. (\%) & 7.7 & 5.7 & & \\
\hline \multirow{3}{*}{$\begin{array}{l}\text { Asymmetric } 1 \times 5 \\
\mathrm{~cm}^{2}\end{array}$} & Opened & 0.795 & 0.710 & 12.0 & \multirow{3}{*}{13.9} \\
\hline & Closed & 0.792 & 0.698 & 13.5 & \\
\hline & Diff. (\%) & 0.4 & 1.7 & & \\
\hline \multirow{3}{*}{$\begin{array}{l}\text { Asymmetric } 1 \times 1 \\
\mathrm{~cm}^{2}\end{array}$} & Opened & 0.677 & 0.629 & 6.5 & \multirow{3}{*}{14.3} \\
\hline & Closed & 0.652 & 0.586 & 11.3 & \\
\hline & Diff. $(\%)$ & 2.8 & 7.3 & & \\
\hline \multirow{3}{*}{$\begin{array}{l}\text { Asymmetric } 2 \times 2 \\
\mathrm{~cm}^{2}\end{array}$} & Opened & 0.886 & 0.844 & 5.0 & \multirow{3}{*}{12.3} \\
\hline & Closed & 0.858 & 0.789 & 8.7 & \\
\hline & Diff. (\%) & 3.3 & 7.0 & & \\
\hline
\end{tabular}

modeled accurately, as its effects are much more important for IMRT treatments than for conventional plans. In our experience, differences found between calculated and measured absolute doses are still too large, even without the fudge factor, so IMRT plans should be managed with caution. Also, errors in the modeling of the output factors for small size segments can lead to important differences in the absolute doses in points affected by the primary x-ray beam of these segments. The overall error we have found in clinical cases is up to $10 \%$ without the proper adjustments to the model. Eliminating the fudge factor decreases the overall error to $3-5 \%$ in most of the clinical cases. Examining the error in absolute doses and in relative distributions for the complete treatment, not for individual fields, will allow us to evaluate the accuracy and safety of each case.

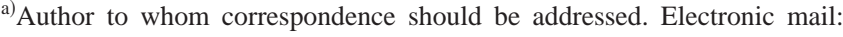
jazcona@unav.es

${ }^{1}$ J. M. Galvin, X. G. Chen, and R. M. Smith, "Combining multileaf field to modulate fluence distributions," Int. J. Radiat. Oncol., Biol., Phys. 27, 697-705 (1993).

${ }^{2}$ T. Bortfeld, D. L. Kahler, T. J. Waldron, and A. L. Boyer, "X-ray field compensation with multileaf collimators," Int. J. Radiat. Oncol., Biol., Phys. 28, 723-730 (1994).

${ }^{3}$ R. A. C. Siochi, "Minimizing static intensity modulation delivery time using an intensity solid paradigm," Int. J. Radiat. Oncol., Biol., Phys. 43, 671-679 (1999).

${ }^{4}$ T. Bortfeld, A. L. Boyer, W. Schlegel, D. L. Kahler, and T. J. Waldron, "Realization and verification of three-dimensional conformal radiotherapy with modulated fields," Int. J. Radiat. Oncol., Biol., Phys. 30, 899-908 (1994).

${ }^{5}$ L. Weber, A. Ahnesjö, P. Nilsson, M. Saxner, and T. Knöös, "Verification and implementation of dynamic wedge calculations in a treatment planning system based on a dose-to-energy-fluence formalism," Med. Phys. 23, 307-316 (1996).
} 
${ }^{6}$ A. Ahnesjö, "Analytic modeling of photon scatter from flattening filters in photon therapy beams," Med. Phys. 21, 1227-1235 (1994).

${ }^{7}$ A. Ahnesjö, "Collimator scatter in photon therapy beams," Med. Phys. 22, 207-278 (1995).
${ }^{8}$ A. Ahnesjö, L. Weber, and P. Nilsson, "Modeling transmission and scatter for photon beam attenuators," Med. Phys. 22, 1711-1270 (1995).

${ }^{9}$ Y. Chen, A. L. Boyer, and C. M. Ma, "Calculation of x-ray transmission through a multileaf collimator," Med. Phys. 27, 1717-1726 (2000). 